\title{
APDP7.pdf \\ 20-fs 1.6-mJ Pulses from a cw-Diode-Pumped Single-Stage 1-kHz Yb Amplifier
}

\author{
Giedrius Andriukaitis ${ }^{1}$, Daniil Kartashov ${ }^{1}$, Audrius Pugžlys ${ }^{1}$, Dušan Lorenc ${ }^{1}$, Andrius Baltuška ${ }^{1}$, \\ Linas Giniūnas², Romualdas Danielius² ${ }^{2}$ Ömer F. Ilday ${ }^{3}$ \\ ${ }^{1}$ Photonics Institute, Vienna University of Technology, Gusshausstrasse 27-387, A-1040, Vienna, Austria \\ ${ }^{2}$ Light Conversion Ltd., P/O Box 1485, Sauletekio Avenue 10, LT-10223, Lithuania \\ ${ }^{3}$ Department of Physics, Bilkent University, Cankaya, Ankara 06800, Turkey
}

\begin{abstract}
J}$ pulses from a fiber-oscillator-seeded DPSS $\mathrm{Yb}_{\mathrm{CaF}} \mathrm{CaOPA}_{2} \mathrm{MO}$ spectrally broadened in Ar and recompressed to 20 fs using a pair of LAK14 prisms. Multimillijoule 12-fs pulses are feasible upon higher-order spectral phase correction.
\end{abstract}

(c) 2010 Optical Society of America

OCIS codes: (320.5520) Pulse compression; (140.3615) Lasers, ytterbium

For over 15 years Ti:sapphire pulse amplifiers emitting $25-150$-fs pulses at $\mathrm{kHz}$ repetition rates have dominated the field of ultrafast applications and ultrashort-pulse laser technology, ultimately leading to the generation of nearsingle-cycle pulses around the wavelength of $800 \mathrm{~nm}$ via external compression. Self-phase modulation (SPM) induced spectral broadening in a gas-filled hollow fiber and laser beam filamentation in gases are well developed techniques for routine production of few-optical-cycle pulses at a sub-mJ energy [1-5]. In this contribution we show encouraging results in millijoule-level pulse broadening and recompression obtained with a novel broadband diodepumped single-stage regenerative 1030-nm Yb:CaF 2 amplifier [6], which holds potential for a robust, compact and cheap alternative to $\mathrm{kHz}$ Ti:sapphire amplifiers, because no additional pump lasers for the seeder and the amplifier are required.

The layout of the system is presented in Fig. 1. In comparison with the performance of our cw-pumped $\mathrm{Yb}$ laser reported earlier [6], the output energy before the grating compressor was scaled up to $5.5 \mathrm{~mJ}$ at $1 \mathrm{kHz}$ as the result of adopting a Brewster-cut $\mathrm{Yb}$ crystal configuration that has replaced our previous AR-coated $\mathrm{Yb}: \mathrm{CaF}_{2}$ slabs. To reduce the system complexity, the deformable mirror in the stretcher is not included, lowering the fidelity of pulse compression and marginally extending the duration form $170 \mathrm{fs}$ to about $200 \mathrm{fs}$. Because of the high optical loss on the diffraction gratings in the compressor, the energy of the compressed pulse is currently limited to $2.5 \mathrm{~mJ}$ at $1030 \mathrm{~nm}$.

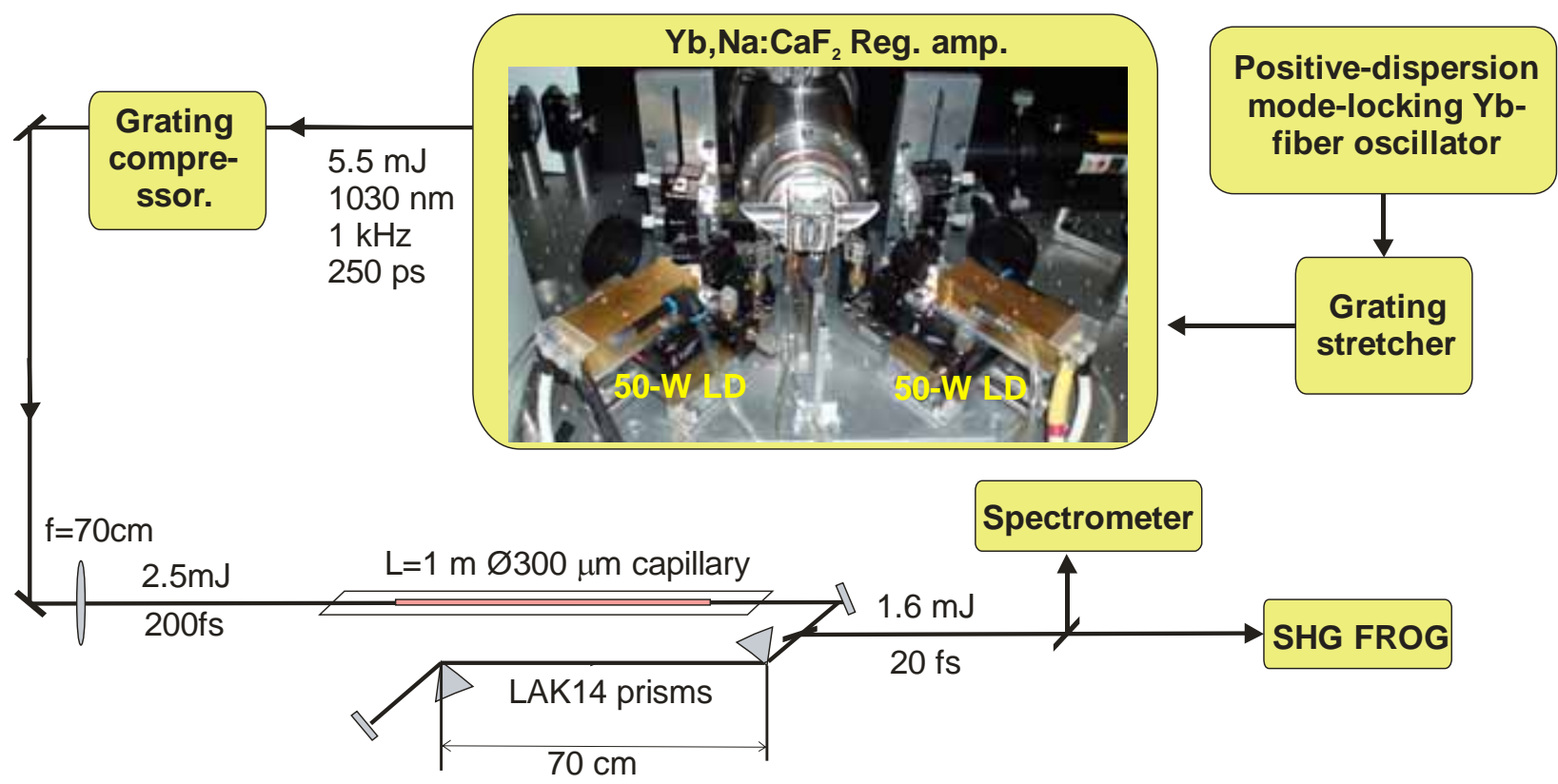

Fig.1: Layout of experimental setup. Inset shows the gain module of the $\mathrm{Yb}$ amplifier pumped with two 50-W cw 977-nm diode bars. 
The output beam of a diffraction-limited quality was coupled into a 300- $\mu \mathrm{m}$ diameter, 1-m-long fused silica hollow-core fiber mounted on a V-groove aluminum holder inside of a vacuum tube with 1-mm-thick Brewster windows. Prior to filling it with gas, the tube is evacuated below $10^{-2} \mathrm{mbar}$. Owing in part to the outstanding input beam quality, the throughput of the hollow fiber was $65 \%(\sim 1.6 \mathrm{~mJ})$ and remained nearly constant with gas pressure. Similarly, high throughput can be maintained within a wide range of input pulse energies (Fig. 2), indicating that the system is further scalable to higher energies even in Argon, because the ionization losses at the wavelength of 1030 $\mathrm{nm}$ are less dramatic than in Ti:sapphire systems operating at $800 \mathrm{~nm}$.

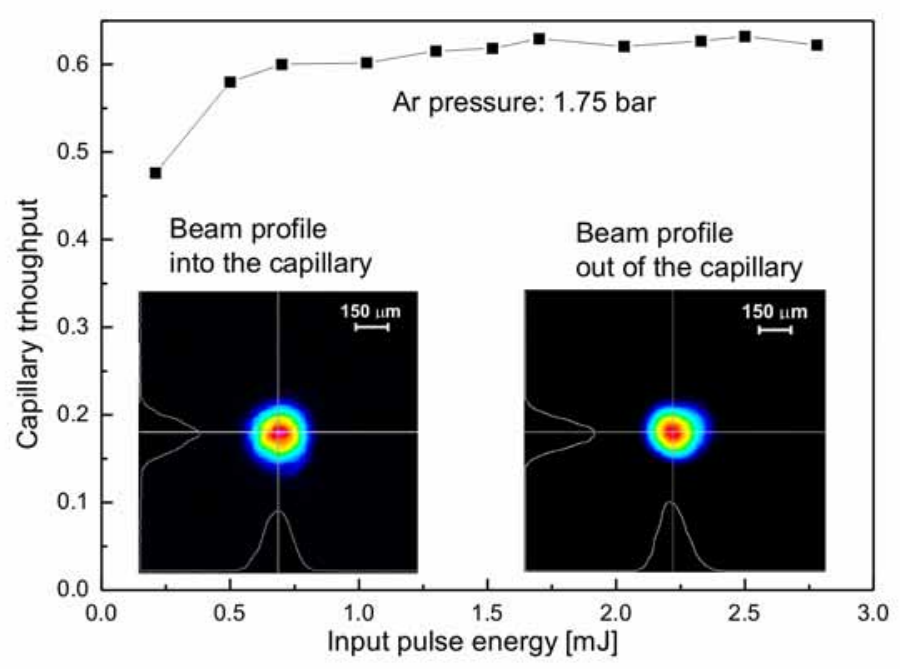

Fig.2: Throughput of a 300- $\mu \mathrm{m}$ Ar-filled capillary at a constant gas pressure (1.75 bar) and input beam focusing conditions $(f=75 \mathrm{~cm})$. Insets show the far-field distribution of the output after the grating compressor (left) and self-phase-modulated beam at the output of the hollow fiber (right). The profiles are measured at the foci of $f=100 \mathrm{~cm}$ lens and $f=200 \mathrm{~cm}$ spherical mirror respectively.

The highest Ar pressure used in our experiments was 2.4 bar, at which point bright visible white light generation was observed and attributed to the onset of filamentation in Ar inside the capillary bore. Spectra supporting a 12-fs transform-limited pulse duration were recorded at 2.2 bar. FROG characterization of the chirped pulses has revealed a reasonably behaved phase to attempt partial pulse compression with a simple prism compressor. We have used a pair of Brewster-angled LAK14 prisms separated by $\sim 70 \mathrm{~cm}$. In the case of Ar pressure of 2.0 bar, the duration of the partially compressed pulse was reduced to $\sim 30$ fs and the carrier wavelength was strongly blue-shifted to 980 $\mathrm{nm}$. The corresponding results of SHG FROG characterization for 2.0 bar are given in Fig. 3. Note that the choice of the working Ar pressure below the optimum value for the broadest spectral broadening is deliberate, in order to fit the pulse spectrum within the effective phase correction bandwidth of the prism pair.
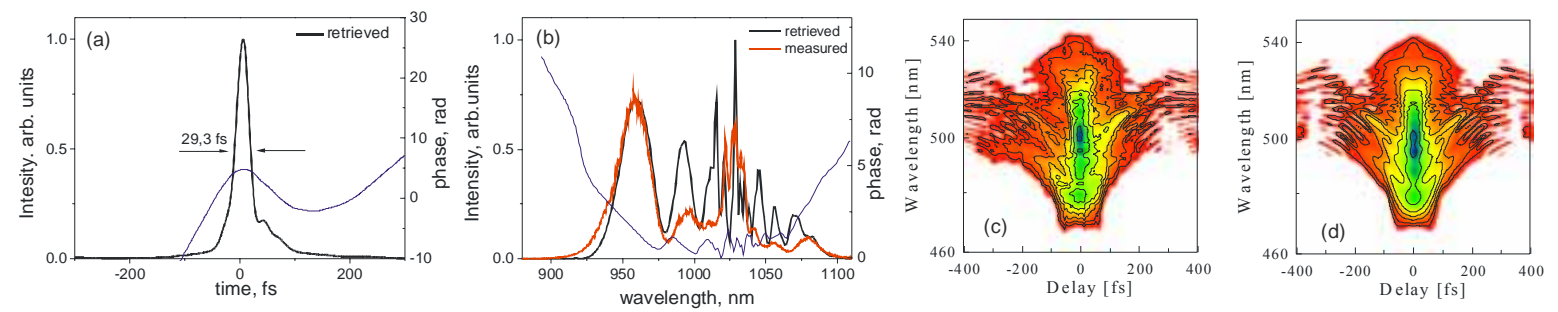

Fig. 3: Partial recompression to $30 \mathrm{fs}$ of strongly blue-shifted 1.6-mJ pulses with a LAK14 prism pair of the pulse transmitted through the hollow fiber at 2.0 bar of Ar. (a) reconstructed temporal intensity and phase. (b) spectral intensity and phase. (c) measured and (d) reconstructed SHG FROG traces.

At a reduced Ar pressure ( 1.75 bar), the spectrum broadens nearly symmetrically around the central wavelength of the injected pulse. In this configuration we have easily achieved pulse recompression to 20.4 fs (15.7 fs Fourier spectrum limit). The results of the corresponding SHG FROG characterization are presented in Fig.4.

The developed system has large potential for scaling up the energy of the compressed pulses. This potential originates from a substantially longer wavelength, in comparison with widely used Ti:Sapphire-based femtosecond laser systems. The intensity of a laser pulse which can be successfully transported and spectrally broadened in a gasfilled hollow fiber is limited by ionization of the gas. In spite of attempts to use the ionization nonlinearity for 
spectral broadening of pulses at a multi-mJ energy level [7,8], SPM in gases is still the most controllable and reliable technique for ultrashort pulse production. The inner diameter of a hollow-core waveguide is limited by coupling and maintaining of the fundamental mode of the waveguide when a laser pulse propagates through. Thus, the maximum energy in a laser pulse which can be realistically transmitted and spectrally broadened for the Ti:Sapphire wavelength of $800 \mathrm{~nm}$ is $\leq 1 \mathrm{~mJ}$ for a $250-300 \mu \mathrm{m}$ capillary filed by Ne. In our 1030-nm system, we expect that a Neon-filled capillary with a diameter of up to $400-450 \mu \mathrm{m}$ can be utilized, which promises a potential increase of the compressed pulse energy by a factor of $4-5$ at least.
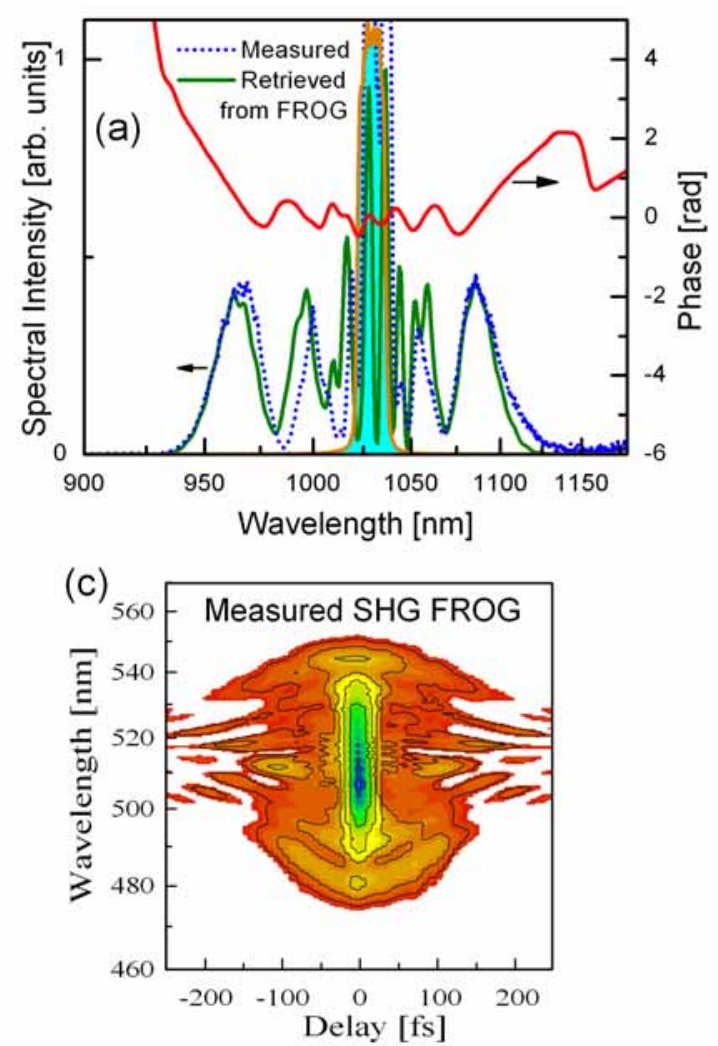
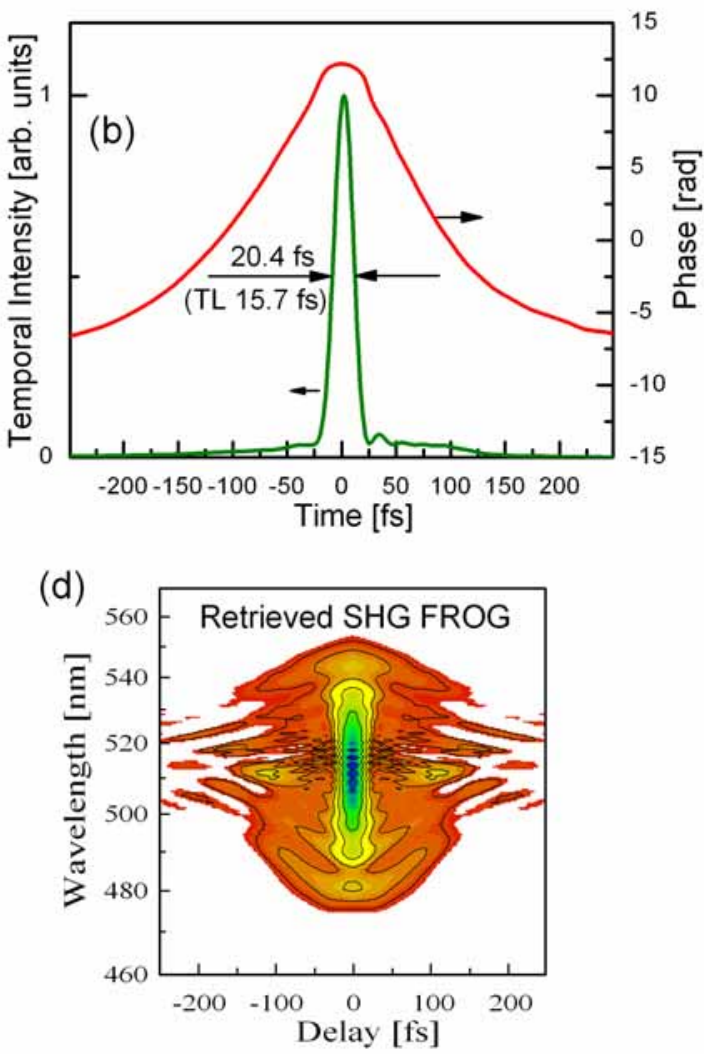

Fig. 4: Partial recompression to 20 fs with a LAK14 prism pair of the pulse transmitted through the hollow fiber at 1.75 bar of Ar. (a) spectral intensity and phase. (b) reconstructed temporal intensity and phase. (c) measured and (d) reconstructed SHG FROG traces. Shaded contour in (a) shows the input pulse spectrum.

In conclusion, we demonstrated for the first time efficient pulse compression at millijoule energy level of the output of a $200 \mathrm{fs}, 1 \mathrm{kHz}$, cw-diode-pumped Yb-based MOPA, using spectral broadening in a gas-filled hollow-core fiber and the simplest prism compressor. This system has large potential for further energy scaling of the compressed pulses and can compete with similar energy level Ti:sapphire laser amplifiers over which, owing to direct diode pumping, it holds the edge in terms of robustness, dependability, and simplicity.

\section{References}

[1] M. Nisoli, S. De Silvestri, and O. Svelto "Generation of high energy 10 fs pulses by a new pulse compression technique", Appl. Phys. Lett. 68, 2793-2795 (1996).

[2] M. Nisoli et al. "Compression of high-energy laser pulses below 5 fs", Opt. Lett. 22, 522-524 (1997).

[3] G. Stibenz, N. Zhavoronkov, and G. Steinmeyer "Self-compression of millijoule pulses to 7.8 fs duration in a white-light filament", Opt. Lett. 31, 274-276 (2006).

[4] C.P. Hauri et al. "Intense self-compressed, self-phase-stabilized few-cycle pulses at $2 \mu \mathrm{m}$ from an optical filament", Opt. Lett. 32, $868-870$ (2007).

[5] A. Zaïr et al. "Spatio-temporal characterization of few-cycle pulses obtained by filamentation", Opt. Exp. 15, 5394-5405 (2007).

[6] A. Pugžlys et al. "Multi-mJ, 200 fs, cw-pumped, cryogenically cooled, Yb, Na:CaF 2 amplifier", Opt. Lett. 34, 2075-2077 (2009).

[7] A. Suda et al. "Generation of sub-10-fs, 5-mJ-optical pulses using a hollow fiber with a pressure gradient", Appl. Phys. Lett. 86, 111116(1-3) (2005).

[8] A. Babin et al. "Compression of High-Intensity Femtosecond Laser Pulses Due to Ionization Self-Phase Modulation in Gas-Filled Capillary Tubes", Las. Phys. 13, 1050-1053 (2003). 